\title{
HL-A8: A Genetic Link between Dermatitis Herpetiformis and Gluten-Sensitive Enteropathy
}

\author{
Stephen I. Katz, Z. Myron Falchuk, Mark V. Dahl, \\ G. Nicholas Rogentine, and Warren Strober \\ From the Dermatology Service, Walter Reed General Hospital, Washington, D. C. 20012, \\ Digestive and Hereditary Diseases Branch, National Institute of Arthritis \\ and Metabolic Diseases, and the Immunology and Metabolism Branches, \\ National Cancer Institute, Bethesda, Maryland 20014
}

A B S T R A C T Histocompatibility antigen HL-A8 was found in $58 \%$ of 26 patients with dermatitis herpetiformis (DH) compared to $24 \%$ of a normal group. This difference in antigen frequency is significant at the $P<0.003$ level. In a previous study, the frequency of this same genetic marker was found to be significantly increased in patients with gluten-sensitive enteropathy (GSE) $(88 \%$ in patients vs. $22 \%$ in controls). The finding of an increased incidence of the HL-A8 antigen in both $\mathrm{DH}$ and GSE supports the concept that these diseases are related and provides a genetic basis for the association between the two.

\section{INTRODUCTION}

Dermatitis herpetiformis $(\mathrm{DH})^{1}$ is frequently associated with duodenal-jejunal villous atrophy similar to that found in gluten-sensitive enteropathy (GSE) $(1,2)$. The basis for this association is unknown. The recent observation by Falchuk, Rogentine, and Strober (3) that $88 \%$ of patients with GSE have histocompatibility antigen HL-A8 led us to determine the HL-A antigen frequencies of patients with $\mathrm{DH}$ to establish whether the frequency of this genetic marker (HL-A8) is increased in these patients as well.

\section{METHODS}

Patients. 26 unrelated Caucasian patients (ages 21-71 yr) were tested. In each patient, the diagnosis of $\mathrm{DH}$ was established by history, physical and histologic examination of the eruption, and prompt improvement in symptoms with sulfone or sulfapyridine treatment. Duration of disease

Received for publication 11 May 1972 and in revised form 30 August 1972.

${ }^{1}$ Abbreviations used in this paper: $\mathrm{DH}$, dermatitis herpetiformis; GSE, gluten-sensitive enteropathy; Ir, immune response. ranged from 1 to $15 \mathrm{yr}$. In each patient a negative indirect immunofluorescent test for basement membrane antibodies excluded the diagnosis of bullous pemphigoid (4). All patients were taking either sulfones or sulfapyridine at the time of HL-A testing. None of the patients had symptoms associated with malabsorption and all had normal serum carotene levels (5).

$H L-A$ typing. HL-A antigens were determined by the use of a lymphocyte microcytotoxicity method as previously described (6). Sera used to detect HL-A antigens were obtained from the Serum Bank maintained by the Transplantation and Immunology Branch of the National Institute of Allergy and Infectious Diseases and from our own collection. Antigens typed for are shown in Table I. In all instances (except W14, W15, and W22, where only one serum was used) at least 2 and as many as 10 different sera were used to define each specificity. An antigen was assigned to an individual when all or nearly all sera specific for the antigen reacted with his cells. The reproducibility of the assay when replicate determinations were done in normal individuals was $97 \%$. The control group consisted of 251 normal Caucasian blood bank donors, except for the antigen W5, where 201 normal blood bank donors were tested.

Statistical methods. The individual HL-A antigen frequiencies in the $\mathrm{DH}$ patients were compared with the antigen frequencies in the control group. $P$ values of differences in frequencies were determined by multiple normal deviate tests (7) which adjust the probability levels by correcting for the number of antigens tested for in each segregant series. Significance was assigned to frequency differences when $P$ values were less than 0.01 .

\section{RESULTS}

$H L-A$ antigens in $D H$ patients. HL-A antigen frequencies of 251 unrelated control individuals, representing a wide spectrum of ethnic and cultural backgrounds, are shown in Table I. These frequencies were determined concurrently with those of the DH patients and are similar to those published by us (3) and others for normal Caucasians (8). 
TABLE I

$H L-A$ Antigen Frequencies in Normal Individuals and Patients with $\mathrm{DH}^{*}$

\begin{tabular}{|c|c|c|}
\hline $\begin{array}{c}\text { HL-A } \\
\text { antigen }\end{array}$ & $\begin{array}{c}\text { Normal } \\
\text { subjects } \\
(251)\end{array}$ & DH (26) $\ddagger$ \\
\hline
\end{tabular}

First segregant series

$\begin{array}{rll}1 & 0.287 & 0.615 \\ 2 & 0.454 & 0.385 \\ 3 & 0.243 & 0.077 \\ 9 & 0.191 & 0.154 \\ 10 & 0.155 & 0.154 \\ 11 & 0.100 & 0.154 \\ \text { W19 } & 0.120 & 0.077 \\ \text { W28 } & 0.076 & 0.077\end{array}$

Second segregant series

$\begin{array}{rll}5 & 0.108 & 0 \\ 7 & 0.235 & 0.077 \\ 8 & 0.239 & 0.577 \\ 12 & 0.219 & 0.346 \\ 13 & 0.040 & 0 \\ W 5 & 0.149 & 0.077 \\ W 10 & 0.143 & 0.115 \\ W 14 & 0.060 & 0.077 \\ W 15 & 0.084 & 0.077 \\ W 17 & 0.032 & 0 \\ W 18 & 0.020 & 0.077 \\ W 22 & 0.032 & 0.077\end{array}$

* The numbers of individuals studied are in parentheses.

$\ddagger$ Differences from normal not significant $(P>0.01)$ except for HL-A1 and HL-A8 where differences were significant at $P<0.005$.

The HL-A antigen frequencies of the DH patients also representing a broad ethnic and cultural background are likewise shown in Table I. The frequency of HL-A8 in patients with $\mathrm{DH}$ was significantly greater $(P<$ 0.003 ) than normal. $58 \%$ of DH patients had HL-A8, whereas this antigen was found in only $24 \%$ of normal individuals. A similar increase in the frequency of HL-A8 was also observed in a group of patients with GSE previously studied: $88 \%$ of GSE patients had HL-A8 whereas this antigen was found in only $22 \%$ of a concurrently studied group of normal subjects (3).

The frequency of a second antigen, HL-A1, was significantly increased in the DH patient group compared to the normal group (62\% frequency vs. $29 \%$ frequency, respectively, $P<0.005)$. The frequency of HL-A1 was also increased in the GSE patient group as compared to the normal group, but not significantly $(P>0.01)$ (3). In assessing the importance of this increase in HL-A1 frequency, it should be noted that $77 \%$ of our normal individuals with HL-A8 also had HL-A1. In ad- dition, this high degree of association between HL-A1 and HL-A8 has been previously noted in other control populations (9). Thus, the increase of HL-A1 in the patients with DH (and possibly GSE) is most likely attributable to the increase in HL-A8.

Each of the GSE patients not having HL-A8 had HL-A12 in the second segregant series (3). This antigen occurred in 5 of $11 \mathrm{DH}$ patients not having HL-A8 (Fig. 1). Thus, $100 \%$ of GSE patients had HL-A 8 or HL-A12 antigens and 77\% of DH patients have these antigens in the second segregant series.

Family studies. The HL-A phenotypes of family members of three patients were determined. The mother of patient S. P. had HL-A8. Children of both patients I. S. and $\mathrm{K}$. H. had HL-A8 whereas this type was not present in either of the spouses. These findings indicate that, in the three patients studied, HL-A8 was an inherited characteristic.

Effect of drug therapy. Each of the patients with $\mathrm{DH}$ was on intermittent sulfone therapy and it was therefore of interest to test the possibility that this drug may induce the HL-A8 phenotype. We therefore studied the HL-A phenotypes of 19 Caucasian patients with leprosy also taking sulfones at a generally higher dosage than the $\mathrm{DH}$ patients. In this patient group the HL-A8 frequency was $6 \%$, if anything lower than the normal frequency.

\section{DISCUSSION}

Histologic intestinal abnormalities similar to those found in the small intestinal mucosa of patients with GSE have been found in more than two-thirds of patients with $\mathrm{DH}$, suggesting the presence of an association between these two diseases $(1,2,10,11)$. However, in contrast to patients with GSE, very few patients with DH have gastrointestinal symptoms or biochemical evidence of malabsorption. Only 2 of our 26 patients had symptoms referable to the gastrointestinal tract and all had normal serum carotene levels.

The association between DH and GSE, though definite, appears to be indirect. Firstly, the mucosal changes observed in patients with $\mathrm{DH}$ are reversible with a gluten-free diet whereas the eruption usually does not respond to this treatment $(1,11,12)$. Conversely, sulfone or sulfapyridine administration is an effective treatment for the rash but does not reverse the intestinal abnormalities in patients with DH or GSE $(1,12)$. Secondly, the skin lesions of $\mathrm{DH}$ show subepidermal blisters with an acute inflammatory infiltrate of neutrophils and eosinophils in the dermal papillae adjacent to the blister. In contrast, the small intestinal lesions in DH and GSE show flattened villi with a chronic inflammatory infiltrate of lymphocytes and plasma cells. These findings indicate that the disease processes in the skin and the gut 
are not characterized by a final common pathway but are related in a more subtle manner, perhaps through an underlying genetic factor which predisposes the individual to both disease processes.

In this study we provide evidence for the existence of such a genetic factor. Patients with GSE have an increased frequency of histocompatibility antigen HL-A8 (88\%) (3). Similarly, patients with $\mathrm{DH}$ also show an increased frequency of this antigen (58\%). Since the enteropathy occurs in about two-thirds of $\mathrm{DH}$ patients $(1,2,10,11)$ one might expect that $88 \%$ of $\mathrm{DH}$ patients with enteropathy will have HL-A8, i.e., that $59 \%$ would have $\mathrm{HL}-\mathrm{A} 8$. This figure agrees well with the observed incidence. It is, therefore, reasonable to infer that both GSE patients and DH patients with GSE share a common genetic factor: the presence of a common histocompatibility antigen. It will be of interest to see if HL-A8 occurs predominantly in those $\mathrm{DH}$ patients with enteropathy and at the normal frequency in those $\mathrm{DH}$ patients without enteropathy.

Assuming that the HL-A8 antigen is a common genetic factor between $\mathrm{DH}$ and GSE, the question remains, how does this factor bring about two widely diverse pathologic processes? The possible answers to this question may take one of two major forms. First, the genes controlling the HL-A antigen may be fortuitously linked to or be identical with "immune response (Ir) genes" which determine whether or not an individual is capable of making an immune response to certain antigens. Such Ir genes controlling responses to simple polypeptides have been noted in animals and in these cases the presence or absence of the gene has been associated with histocompatibility type (13). In both DH and GSE there is considerable evidence that immunologic mechanisms are important and primary pathogenetic factors $(14,15$, 16). For example, in $\mathrm{DH}, \operatorname{IgA}$, and occasionally $\operatorname{IgG}$ and complement are found deposited in a characteristic granular pattern near the basement membrane adjacent to skin lesions $(17,18,19)$. Similarly, in GSE, patients respond to gluten challenge with an increase in mucosal $\operatorname{IgA}$ and $\operatorname{IgM}$ synthesis, $40-80 \%$ of which is antigliadin antibody (16). These immunologic phenomena could be under the control of an Ir gene which is in some way related to the $\mathrm{HL}-\mathrm{A} 8$ histocompatibility gene.

Secondly, HL-A antigens are present on the surface of many different cells and may, therefore, function as receptors capable of binding infectious agents or other materials of pathologic significance. Thus, in GSE the pathologic process may involve the attachment of gliadin to epithelial surfaces (20). which then results in direct tissue injury or injury mediated by an immunologic reaction. In the same way, a yet unknown infectious agent or nonliving material may bind to skin sites and initiate the set of events causing the eruption of $\mathrm{DH}$.

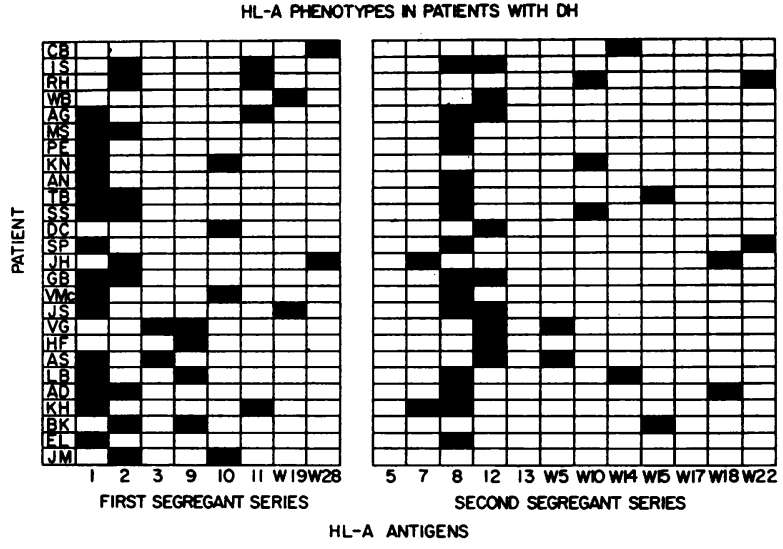

Figure 1 HL-A phenotypes in patients with DH.

Even if one of these possibilities is correct, the HL-A8 gene cannot be the only factor involved in the pathogenesis of these diseases. HL-A8 is not uncommon in a normal Caucasian population (24\% frequency) and most people with HL-A 8 have neither DH nor GSE. Despite this, the finding of an increased incidence of HL-A8 phenotype in these two diseases constitutes additional evidence that $\mathrm{DH}$ and GSE are associated and provides a genetic basis for the link between the two.

\section{ACKNOWLEDGMENTS}

We wish to thank Dr. Robert Trapani of Microbiological Associates for his assistance in conducting the HL-A typings and the physicians on the Dermatology Service at Walter Reed General Hospital and the many members of the D. C. Dermatological Society for referring us many of the patients in this study. In addition, we wish to thank the physicians and staff of the Public Health Service Hospital at Carville, La. and the Communicable Disease Center at Atlanta, Ga. for allowing us to study the patients with leprosy.

\section{REFERENCES}

1. Shuster, S., A. J. Watson, and J. Marks. 1968. Coeliac syndrome in dermatitis herpetiformis. Lancet. 1: 1101.

2. Brow, J. R., F. Parker, W. M. Weinstein, and C. E. Rubin. 1971. The small intestinal mucosa in dermatitis herpetiformis. I. Severity and distribution of the small intestinal lesion and associated malabsorption. Gastroenterology. $60: 355$.

3. Falchuk, Z. M., G. N. Rogentine, and W. Strober. 1972. Predominance of histocompatibility antigen $\mathrm{HL}-\mathrm{A} 8$ in patients with gluten-sensitive enteropathy. J. Clin. Invest. $51: 1602$.

4. Katz, S. I., K. Halprin, and T. M. Inderbitzin. 1969. The use of human skin for the detection of anti-epithelial auto antibodies. A diagnostic and prognostic test. J. Invest. Dermatol. 53: 390.

5. Varley, H. 1962. In Practical Clinical Biochemistry Heinemann and Zsolnay Ltd., London. 3rd edition. 509.

6. Mittal, K. K., M. R. Mickey, D. P. Singal, and P. I. Terasaki. 1968. Serotyping for homotransplantation. XVIII. Refinement of microdroplet lymphocyte cytotoxicity test. Transplantation. 6: 913.

Histocompatibility Antigen in DH and GSE 
7. Wilks, S. S. 1962. In Mathematical Statistics. John Wiley and Sons, Inc., New York. 290.

8. Dausset, J., J. Columbani, L. Legrand, and M. Fellous. 1970. Genetics of the HL-A system. Deduction of 480 Haplotypes. In Histocompatibility Testing 1970. P. I. Terasaki, editor. Munksgaard A/S, Copenhagen. 53.

9. Albert, E. D., M. R. Mickey, and P. I. Terasaki. 1971. Genetics of 4 new HL-A specificities in Caucasian and Negro population. Transplant. Proc. 3: 95.

10 Marks, R. 1968. Dermatitis herpetiformis and the gastrointestinal tract. Trans. St. John's Hosp. Dermatol. Soc. 54: 141.

11. Fry, L., P. Keir, R. M. H. McMinn, J. D. Cowan, and A. V. Hofbrand. 1967. Small intestinal structure and function and haematological changes in dermatitis herpetiformis. Lancet. 2 : 729.

12. Weinstein, W. M., J. R. Brow, F. Parker, and C. E. Rubin. 1971. The small intestinal mucosa in dermatitis herpetiformis. II. Relationship of the small intestinal lesion to gluten. Gastroenterology. 60: 362.

13. McDevitt, H. O., and B. Benacerraf. 1969. Genetic control of specific immune responses. Adv. Immunol. 11:31.

14. Editorial. 1972. Dermatitis herpetiformis-a disorder of immunity? Lancet. 1: 621 .
15. Loeb, P. M., W. Strober, Z. M. Falchuk, and L. Laster. 1971. Incorporation of L-leucine- ${ }^{14} \mathrm{C}$ into immunoglobulins by jejunal biopsies of patients with celiac sprue and other gastrointestinal diseases. J. Clin. Invest. 50: 559.

16. Falchuk, Z. M., L. Laster, and W. Strober. 1971. Gluten sensitive enteropathy: intestinal synthesis of antigluten antibody in vitro. Clin. Res. 19: 390. (Abstr.)

17. van der Meer, J. B. 1969. Granular deposits of immunoglobulins in the skin of patients with dermatitis herpetiformis: an immunofluorescent study. $B r . J$. Dermatol. $81: 493$.

18. Chorzelski, T. P., E. H. Beutner, S. Jablonska, M. Blaszcyk, and C. Triftshauser. 1971. Immunofluorescence studies in the diagnosis of dermatitis herpetiformis and its differentiation from bullous pemphigoid. J. Invest. Dermatol. 56: 373.

19. Cormane, R. H. 1970. Revised immunofluorescence interpretations of skin in lupus erythematosus (LE) and dermatitis herpetiformis. Int. Arch. Allergy Appl. Immunol. $39: 107$.

20. Rubin, W., A. S. Fauci, M. H. Sleisenger, and G. H. Jeffries. 1965. Immunofluorescent studies in adult celiac disease. J. Clin. Invest. $44: 475$. 